\title{
3 Research Suare

\section{Survival of patients with appendiceal neuroendocrine tumors: whether they benefit from right hemicolectomy?}

\section{Haiping Lin}

Zhejiang University School of Medicine https://orcid.org/0000-0003-3054-9021

\section{Liyuan Wang}

Zhejiang University School of Medicine

\section{Yang Bai}

Zhejiang University School of Medicine

Chenyang Ge

Zhejiang University School of Medicine

\section{Hongjuan Zheng}

Zhejiang University School of Medicine

\section{Shian Yu ( $\nabla$ zjjhysa@163.com )}

Zhejiang University School of Medicine https://orcid.org/0000-0002-6326-7410

\section{Research}

Keywords: appendiceal neuroendocrine tumors, Fine-Gray proportional hazards model, metastatic lymph nodes, right hemicolectomy, appendectomy

Posted Date: November 12th, 2020

DOI: https://doi.org/10.21203/rs.3.rs-24718/v2

License: (c) (1) This work is licensed under a Creative Commons Attribution 4.0 International License. Read Full License 


\section{Abstract}

Background: Current guidelines recommend right hemicolectomy for appendiceal neuroendocrine tumors (A-NETs) patients with lymph node ( $L N)$ metastasis. However, prognosis of these patients is favorable, and the prognostic impact of metastatic $L N$ is controversial.

Objective: The study aims to evaluate the prognostic factors of A-NETs, and explore whether right hemicolectomy/ more extended procedure (RHCM) improves prognosis compared to less extended than right hemicolectomy (LRHC).

Methods: Patients with A-NETs were identified from the Surveillance, Epidemiology, and End Results (SEER) database. The Fine-Gray proportional hazards model was established to calculate subdistribution hazard ratios of prognostic factors. A propensity score matching was performed to balance intergroup differences between the LRHC and RHCM groups, and survival difference between the after-matched groups was tested using the Gray test. Subgroup analyses were also conducted.

Results: In the multivariate analysis, histological type and distant metastatic status were associated with prognosis, while tumor size and nodal status were not. After propensity score matching, the patients' characteristics were well balanced. RHCM did not confer survival benefits in the whole after-matched patients or any subgroup.

Conclusions: Metastatic LN does not significantly impact prognosis, and RHCM fails to improve prognosis compared to LRHC. Therefore, the current "LN-decided" surgical procedure may not be suitable for patients with A-NETs.

\section{Introduction}

Appendiceal neuroendocrine neoplasms (A-NENs) are rare, but they represent the most common tumor of the appendix, and their incidence has increased moderately in recent years[1-4]. Different from other neuroendocrine neoplasms, A-NENs are rarely accompanied with carcinoid syndrome, and their discovery is usually accidental after surgery for acute appendicitis, with around $0.5 \%$ of all appendectomies[5].

Some studies have reported lymph node ( $L N)$ metastasis is a hallmark of aggressive behavior and a predictor for poor prognosis, while others have reported favorable prognosis even in patients with LN metastasis, raising doubts about the prognostic value of metastatic $\operatorname{LNs}[1,6,7]$. However, previous studies mostly included patients with both well and poor differentiated A-NENs, the former was classified into appendiceal neuroendocrine tumors (A-NETs) and the latter appendiceal neuroendocrine carcinomas (A-NECs) in the 2019 WHO Classification of Digestive System Tumors, without considering different biological characteristics between these two types $[6,8]$. It has been proved that the A-NETs are of indolent characteristic and favorable prognosis compared to the A-NECs $[9,10]$. 
Oncological right hemicolectomy is suggested for patients with LN metastasis or suspected LN metastasis, such as tumor size $\geq 2 \mathrm{~cm}$, to eliminate tumor dissemination[1, 11]. However, many studies now argue that this "LN-decided" surgical procedure fails to improve outcome[12-16]. Previous studies are mainly retrospective studies and case series. Considering the rarity and favorable prognosis of ANETs, a randomized trial is difficult to conduct because of the enormous sample size required and the long follow-up period needed. Clinicians sometimes face dilemma that whether or not to perform a reoperation or an extended operation after initial appendectomy[12, 14].

Using a large, population-based database, our study focuses on A-NETs rather than A-NECs or the mixture of both. We aim to evaluate the prognostic factors, especially LN status, of A-NETs, and explore whether right hemicolectomy or more extended surgery improves prognosis compared to less than right hemicolectomy.

\section{Methods}

\section{Availability of data and materials section}

The Surveillance, Epidemiology, and End Results (SEER) database collects information on cancer from registries sponsored by the US National Cancer Institution. In current, the SEER database consists of population-based cancer registries that cover $34.6 \%$ US population. The database collects data (e.g., patient demographics, primary tumor location, tumor morphology, diagnosis, and first course of treatment) and tracks the life status of patients.

The permission to access the database was obtained with the reference number 12005-Nov2019. The SEER database is an open-access cancer database that only contains deidentified patient data. Therefore, this study was exempted from the approval of the review board of the Affiliated Jinhua Hospital, Zhejiang University School of Medicine. Patients with A-NETs from 2000 to 2015 were identified by using the SEER Stat software. Patients diagnosed before 2000 were excluded because some variables were not collected on the database until 2000; patients diagnosed after 2015 were excluded to ensure adequate follow-up period.

\section{Inclusion and Exclusion Criteria}

Patients met the following criteria were included: age of 18-80 years old; underwent curative-intend surgery (surgery of primary site codes 30-80); tumor site ICD-0-3 code 18.1 (appendix); histology ICD-0-3 codes 8240 (carcinoid tumor, malignant), 8241 (enterochromaffin cell carcinoid), 8242 (enterochromaffinlike cell tumor, malignant), 8244 (composite carcinoid), 8245 (adenocarcinoid tumor), 8246 (neuroendocrine carcinoma), 8249 (atypical carcinoid tumor) (Goblet cell carcinoids were not included because it is now recognized to have a minor neuroendocrine component, and its classification changes to goblet cell adenocarcinoma in the 2019 WHO classification of tumors of the digestive system[17]); well or moderately differentiated grade (A-NETs). 
Patients met the following criteria were excluded: patients with incomplete documentation, such as tumor size and LN status, were excluded; patients with multiple primary tumors were excluded to eliminate the survival impact from other tumors; patients with survival time less than 1 month were excluded because these patients are at risk of death of perioperative complications.

\section{Data collection}

The age and year at diagnosis, gender, race, histological type, differentiated grade, tumor size, depth of invasion, status of LNs and distant metastasis, surgical procedure, causes of death and survival months were retrieved from the SEER database. Race was classified into white, black and other. Histological type was classified into pure and mixed, and the former included malignant carcinoid tumor, enterochromaffin cell carcinoid, neuroendocrine cell carcinoma and the latter included composite carcinoid, adenocarcinoid tumor, atypical carcinoid tumor. Differentiated grade was classified into well and moderate differentiation. As previous studies reported, depth of invasion was obtained by combining the data of the collaborative stage and the extent of disease, resulting in three categories: invasion of the lamina propria (LP), invasion or through the muscularis propria (MP/TMP), or invasion through the serosa and adjacent structures (TS).[18]. LN status was categorized into no lymph nodes examined (NLNE), lymph nodes examined with negative lymph nodes (LNN) and lymph nodes examined with positive lymph nodes (LNP). According to "RX Summ - Surg Prim Site" values in the database, surgical procedure was divided into two categories: right hemicolectomy/ more extended procedure (RHCM) and less extended than right hemicolectomy ( $\mathrm{LRHC}$ ). The RHCM involves removing the cecum, the total ascending colon, the hepatic flexure, a portion of the transverse colon, and part of the terminal ileum, along with fat and lymph nodes (the exact number of retrieved LNs is not defined because of the characteristic of our population-based study and non-consensus of specific LN number in current guidelines), or more extended surgery; the extent of the LRHC is lesser than the RHCM, including appendectomy, ileocolectomy, and partial and segmental colon resection.

\section{Data analysis}

Demographic and clinical characteristics of the cohort were reported as medians with interquartile ranges (IQR) for continuous variables, and frequencies for categorical variables. Continuous variables were compared using Student $t$ test or Mann-Whitney $U$ test. Categorical variables were compared using chisquared test or Fisher exact test.

The prognosis of patients with well and moderately differentiated A-NETs is favorable, and the incidence of non-cancer specific death (non-CSD) cannot be simply ignored when conducting a survival analysis[19]. Traditional Cox proportional hazard models only considered two statuses of outcome (e.g. alive and death), while competing risk models considered the presence of competing events (e.g. nonCSD). Thus, the competing risk model, rather than the Cox proportional hazard model, was applied in the study. 
The time to cancer specific death (CSD) was calculated from the date of diagnosis to the date of death of cancer; the time to non-CSD was calculated from the date of diagnosis to the date of death of other causes. CSD was regarded as the outcome event, and non-CSD was regarded as the competing event. Univariate and multivariate analyses were conducted. In the univariate analyses, the cumulative incidences of CSD were calculated, and the differences were tested using the Gray tests. Variables significant in the univariable analyses then entered into the multivariable analysis. In the multivariate analysis, subdistribution hazard ratios (SHRs) were calculated to predict the association of variables with CSD (patients with tumor invasion of LP were excluded in both univariate and multivariate analyses because none of them dead of CSD).

To reduce biases from confounders and achieve balance between the RHCM and LRHC groups, a propensity score matching was performed. Based on demographic and clinical characteristics (i.e., age, gender, race, histological type, differentiation, tumor size, depth of invasion and status of LNs), patients were matched with a 1:1 ratio using the nearest neighbor method (caliper set to 0.05 )[20]. Absolute standardized differences were calculated to evaluate pre- and after-matched balance, and a "love plot" was plotted to present them[21]. The differences less than $10 \%$ support intergroup balance, and $0 \%$ is considered no bias. In the after-matched patients, the univariate analysis between the RHCM and LRHC groups was conducted to identify whether RHCM rendered a survival benefit compared to LRHC.

In addition, subgroup analyses of the before-matched patients stratified by age, gender, race, histological type, differentiation, tumor size, nodal status, depth of invasion and metastatic status were conducted to explore whether RHCM improved outcome in a certain group, and a "forest plot" were plotted to present the results. Considering some patients did not undergo lymphadenectomy or had no LNs retrieved, another subgroup analysis (univariate and multivariate competing risk analyses) was conducted in patients with LNs retrieved.

The difference was considered to be statistically significant when the 2-side P-value was less than 0.05 . $R$ software (version 3.6) was applied for data analysis, and R packages survival, survminer, forestplot and cobalt were used.

\section{Results}

\section{Patient characteristics}

In total, 901 patients were identified from the SEER database, with 658 patients undergoing LRHC and 243 patients undergoing RHCM. In the LRHC group, over a half of (68.39\%) A-NETs were less than $10 \mathrm{~mm}$, while only $9.57 \%$ were more than $20 \mathrm{~mm}$; in the RHCM group, nearly a half $(46.09 \%)$ of A-NETs were more than $20 \mathrm{~mm}$, while only $22.22 \%$ were less than $10 \mathrm{~mm}$. Only $20.97 \%$ of the patients underwent lymphadenectomy when they were performed LRHC, and most of the retrieved LNs were negative. In comparison, most patients $(91.77 \%)$ underwent lymphadenectomy in the RHCM group, and a third of the group (33.33\%) had metastatic nodes (Table 1). In patients proved to have negative LNs, $56.57 \%$ 
(142/251) underwent RHCM, while in patients proved to have metastatic LNs, 73.64\% (81/110) underwent it.

Intergroup differences indicated clinicopathological characteristics were not balanced between the two groups. In the RHCM group, A-NETs were poorer differentiated grade, deerper invasion, and had higher proportions of LN and distant metastasis.

\section{Survival analysis}

The endpoint date of follow-up was November 2019, and the median follow-up duration was 148 months (IQR: 138-167). In total, 14 patients (1.55\%) and 18 patients (2.00\%) suffered from CSD and nonCSD, respectively (Figure 1). Note that more patients suffered from non-CSD than CSD (13 patients suffered from non-CSD versus 6 patients suffered from CSD) in the LRHC group, while there were more CSD than non-CSD in the RHCM group (8 patients suffered from CSD versus 5 patients suffered from non-CSD).

In univariate analyses, age, histological type, differentiated grade, tumor size, depth of invasion, status of LNs and distant metastasis were all significantly associated with CSD (Table 2). In patients undergoing lymphadenectomy (361 patients), the patients with LNP had worse prognosis than those with LNN, and the 10 -year CSD cumulative incidences was $0.96 \%$ in former while there was no CSD happened in latter during the 10-year period $(P=0.54)$.

Surgical procedure, which did not reach statistical significance in the univariate analysis, also entered into the next multivariate analysis to explore the surgical impact on survival when controlling confounders. The surgical procedure and the significant variables in the univariate analyses then entered into multivariate analysis. Only histological type and status of distant metastasis kept statistically significant in the multivariate analysis ( $\mathrm{P}$ all $<0.01$ ), while surgical procedure, $\mathrm{LN}$ status and tumor size were not (Table 2).

\section{Subgroup analyses}

Results of subgroup analyses of the before-matched patients were shown in the forest plot (Figure 2). In most subgroups, the surgical procedure was not associated with CSD, and the RHCM was even associated with poorer prognosis in the $41-60$ age subgroup $(\mathrm{SHR}=5.55 ; 95 \% \mathrm{Cl}=1.16-26.60 ; \mathrm{P}=0.03)$ and the no distant metastasis subgroup $(\mathrm{SHR}=7.01 ; 95 \% \mathrm{Cl}=1.45-33.80 ; \mathrm{P}=0.02)$. Only in the distant metastasis subgroup, the RHCM was associated with better prognosis $(\mathrm{SHR}=0.21 ; 95 \% \mathrm{Cl}=0.05-0.95 ; \mathrm{P}$ $=0.04)$.

Results of subgroup analyses of the patients with LNs retrieved (361 patients) were shown in Appendix Table 1. Age, race, histological type and distant metastasis were significantly associated with CSD in the univariate analyses, and entered into the multivariable analysis (Surgical procedure did not reach statistical significance in the univariate analysis, but it also entered into the multivariate analysis). The results of the entire cohort and the subgroup of patients with LNs retrieved are consistent. Histological 
type and status of distant metastasis kept statistically significant in the multivariate analysis $(P=0.01$ and 0.02 , respectively), while surgical procedure, $\mathrm{LN}$ status and tumor size were not.

4. Patient characteristics and survival analyses after propensity score matching

Patients in the LRHC and RHCM groups were matched 1:1, and there were 136 patients in each aftermatched group. Table 3 showed the demographic and clinical characteristics after matching, and $P$ values of each variable were more than 0.05. Appendix Figure 1 showed the total absolute standardized difference was less than $10 \%$, although the absolute standardized differences of some variables were more than $10 \%$. In the after-matched patients, there was no CSD happened in both LRHC and RHCM groups in the 5-year follow-up period, and the 10-year CSD cumulative incidences were $0.00 \%$ in the LRHC group and $0.75 \%$ in the RHCM group, and there was no significant difference of CSD between the two groups $(P=0.67)$ (Appendix Figure 2).

\section{Discussions}

In the population-based study, we proved that the prognosis of A-NETs was extremely favorable, and the impact of non-CSD should be emphasized. In survival analysis, histological type and status of distant metastasis were associated with prognosis, while tumor size and LN status were not.

Right hemicolectomy or extended surgery failed to render survival benefits compared to less than right hemicolectomy, even in the subgroups of patients with metastatic $L N$ and with $\geq 2.0 \mathrm{~cm}$ tumor size.

A-NENs are tumors of heterogeneous entities, and prognoses are various among different types[22]. Previous studies have shown that well and moderate A-NENs, namely A-NETs, are of indolent clinical course and the prognosis of them is favorable, which was proved in our study with only $1.55 \%$ patients suffered from CSD in the entire cohort. In addition, our study found non-CSD made up a similar proportion (2.00\%) as CSD. To our knowledge, there are only one study reported the impact of non-CSD in patients with appendiceal cancer[23]. The study of Jingjing Wu et al. focused on the entire appendiceal cancer and variables associated with non-CSD, while our study paid attention to the A-NETs and variables associated with CSD. Our results suggested non-CSD should be considered in clinical situations. For example, when making clinical decisions for patients who are old or accompanied with severe underlying diseases, appendectomy, rather than right hemicolectomy, seems to be more suitable. It was also the reason why our study applied the competing risk model, rather than the Cox proportional hazard model, because the latter underestimates the cumulative incidences of $\operatorname{CSD}[24,25]$.

Histological type is associated with prognosis and serves as an important predictor for survival[16]. Hsu $C$ et al. compared characteristics and outcomes of malignant carcinoid tumor, goblet cell carcinoid and composite goblet cell carcinoid-adenocarcinoma from the American National Cancer Database, and found malignant carcinoid tumor had the best prognosis and composite goblet cell carcinoidadenocarcinoma had the worst [22]. However, with deeper understanding of goblet cell carcinoid, it was classified to goblet cell adenocarcinoma in the 2019 WHO classification of tumors because of its minor 
neuroendocrine component[17]. Therefore, we excluded goblet cell adenocarcinoma in our study, and also confirmed the prognosis of pure A-NETs was much better than the mixed type. Distant metastasis is another well-established predictor for poor prognosis, and our study found MRHC only rendered survival benefits in the subgroup of distant metastasis.

Tumor size and LN status used to be regarded as main prognostic features of A-NETs. Tumor size $\geq 2$ $\mathrm{cm}$ is the indicator for right hemicolectomy in both the National Comprehensive Cancer Network (NCCN) and the ENETS guidelines because the risk of LN metastasis increases dramatically in $\geq 2 \mathrm{~cm}$ A-NETs[1, 11]. However, neither the tumor size nor the nodal status was significantly associated with CSD when controlling confounders in our study, and there are some studies supporting our findings. Rault-Petit et al. conducted a national study including 403 patients with nonmetastatic A-NETs, 26 of whom was surgically proved to have metastatic nodes, and only 1 patient had recurrence during the follow-up[6]. Recently, Daskalakis et al. conducted a meta-analysis including A-NENs patients, finding there was no significant difference in disease-specific survival between patients with and without lymph node metastasis (10-year disease-specific survival: $99.2 \%$ in patients without LN metastasis versus $95.6 \%$ in patients with LN metastasis)[26].

On the other hand, results of some previous studies are different from our findings, and suggest metastatic LN is the predictor for poor outcome. Note that these previous studies included patients with both A-NETs and A-NECs[27]. When focusing on patients with well differentiated A-NETs, some previous studies reported favorable prognosis even in LN metastasis patients. Lambert et al. reported 114 children and adolescents with well differentiated A-NETs, some of who had metastatic nodes (the exact number was not provided), and all patients were alive and disease free at the last follow up[7]. Combining results of ours and previous studies, we concluded that metastatic LNs should not be regarded as a significant predictor for poor prognosis in patients with A-NETs.

In 1987, Moertel et al. studied 150 patients with pure appendiceal carcinoid tumors, and found recurrences occurred very late and only happened to patients with $\geq 2 \mathrm{~cm}$ tumors[28]. Therefore, they suggested right hemicolectomy was appropriate only for young patients with $\geq 2 \mathrm{~cm}$ tumors. As mentioned in the Background section, current guidelines for A-NETs were "LN-guided". According to current guidelines, RHCM is routinely suggested for patients with LN metastasis or high probabilities of LN metastasis, such as vascular or lymph vessel invasion and mesoappendiceal infiltration $>3 \mathrm{~mm}$, to eliminate metastatic lesions and achieve long-term survival. However, our study suggested RHCM failed to render survival advantage compared to LRHC in both univariate and multivariate analyses. Our finding is supported by results of some previous studies. Crown et al. analyzed a multi-center cohort (61 patients) and found more radical surgical procedures, such as colon resection, did not improve long-term outcomes[12]. Bamboat et al. conducted a retrospective review of 48 appendiceal carcinoid patients, and they failed to find right hemicolectomy confer a prognostic benefit over appendectomy for tumors greater than $2.0 \mathrm{~cm}[29]$. But, as far as we know, there has been no high-quality evidence comparing the prognostic benefits between LRHC and RHCM. 
Considering the low incidence and long-term survival of A-NENs, it is difficult to conduct a randomized controlled trial to compare survival advantage between LRHC and RHCM. In our study, a propensity score matched analysis was performed to achieve intergroup balance, and surprisingly, we failed to observe survival advantage of RHCM over LRHC in the after-matched patients. Lukas Marti et al. hypothesized the reason for non-benefit of RHCM in appendiceal carcinoma was that LNs metastasis was limited, and ileocaecal resection was sufficient for removing metastatic LNs[13]. But his hypothesis was based on LN metastatic patterns in colon cancer patients and needs further confirmation, and we hypothesized that this non-benefit is "blamed" for the current "LN-decided" surgical strategy. Results of the subgroup analyses that patients with positive LNs failed to benefit from the RHCM further strengthened this hypothesis.

On the other hand, colon resection has been reported to increase operation time, blood loss, postoperative complication rates and length of stay, and the postoperative complications such as anastomotic leakage will have a negative impact on both short-term and long-term outcomes[12, 30]. Reoperation also serves as an option for patients with local or distant recurrence during the follow-up period, and previous studies reported patients underwent reoperation could achieve long-term survival[7]. Thus, we concluded that LRHC, such as ileocaecal resection or appendectomy, was curative for A-NETs with LN metastasis, and MRHC did not improve prognosis. In addition, long follow-up term is vital for A-NET patients because we observed happenings of CSD in both RHCM and LRHC groups even after 10 years.

Although our study is based on large population in the USA, it is also characterized by the following limitations. First, the SEER database does not contain records of vascular or lymph vessel invasion, margin states, Ki-67 index and mitotic rate, which may be associated with prognosis. Also, information about post-operative complications, which has been reported to have an influence on survival, is not available in the database. Moreover, the treatment records are incomplete and there is no detailed information regarding chemotherapy and radiotherapy. Finally, this study is a retrospective study and biases such as selection bias and treatment bias are inevitable in our study.

\section{Conclusion}

In conclusion, the study has demonstrated the prognosis of A-NETs is extremely favorable, and non-CSD should be taken into consideration when making clinical decisions. Current guidelines suggest performing RHCM for patients with LN metastasis or suspected LN metastasis, while in our study, LN metastasis was proved not to be associated with poor outcome in A-NETs. Therefore, it indicates the current "LN-decided" surgical strategy was inappropriate, and LRHC was sufficient for patients with ANETs, regardless of nodal status.

\section{Clinical Practice Points}

- The prognosis of well and moderately differentiated A-NETs is extremely favorable, and non-cancer specific death should be taken into consideration when making clinical decisions 
- Age, race, histological type and status of distant metastasis were associated with prognosis in the Fine-Gray proportional hazards model, while tumor size and LN status were not.

- In our study, $42.34 \%$ and $73.21 \%$ patients underwent right hemicolectomy or extended surgery in the negative lymph node group and positive lymph node group respectively, proving current guidelines are "LN-decided".

- Right hemicolectomy or extended surgery failed to render survival benefits compared to less extended than right hemicolectomy, indicating current "LN-decided" surgical strategy is inappropriate.

\section{Declarations}

Acknowledgements

None

Author's contributions

Haiping Lin: Study concept and design; collection, analysis, and interpretation of data; writing of the manuscript; and submission of the manuscript for publication. Liyuan Wang: Collection, analysis, and interpretation of data; writing of the manuscript. Yang Bai, Chenyang Ge, Hongjuan Zheng: Collection, analysis, and interpretation of data; writing of the manuscript. Shian Yu: Study concept and design; revision of the manuscript for important intellectual content; final approval for submission of the manuscript for publication.

\section{Funding}

The study was supported by key program of the Jinhua Municipal Science \& Technology Bureau (grant numbers 2018-3-001a), and key program of the Zhejiang medical and health science \& technology project (grant numbers 2018244976).

Availability of data and materials

The data sources of the manuscript are derived from the de-identified Surveillance, Epidemiology, and End Results (SEER) database (SEER ID: 12005-Nov2019).

Ethics approval and consent to participate

The SEER database is an open-access cancer database that only contains deidentified patient data. Therefore, this study was exempted from the approval of the review board of the Affiliated Jinhua Hospital, Zhejiang University School of Medicine.

Consent for publication

No applicable 
Competing interests

The authors declare that they have no competing interests.

Author details

${ }^{1}$ Zhejiang University School of Medicine, 268 Kaixuan Road, Hangzhou 310000, Zhejiang Province, China.

2 Department of Hepatobiliary Surgery, the Affiliated Jinhua Hospital, Zhejiang University School of Medicine, 365 East People Road, Jinhua 321000, Zhejiang Province, China.

\section{References}

1. Pape, U.F., et al., ENETS Consensus Guidelines for Neuroendocrine Neoplasms of the Appendix (Excluding Goblet Cell Carcinomas). Neuroendocrinology, 2016. 103(2): p. 144-52.

2. JB, M. and B. G, Carcinoid tumors of the gut. Our experience over three decades and review of the literature. Journal of clinical gastroenterology, 1993. 16(2): p. 123-9.

3. Mo, S., et al., Epidemiology of and prognostic factors for appendiceal carcinomas: a retrospective, population-based study. Int J Colorectal Dis, 2019. 34(11): p. 1915-1924.

4. Fraenkel, M., et al., Epidemiology of gastroenteropancreatic neuroendocrine tumours. Best Pract Res Clin Gastroenterol, 2012. 26(6): p. 691-703.

5. MB, N., et al., Gastroenteropancreatic neuroendocrine tumours: the current incidence and staging based on the WHO and European Neuroendocrine Tumour Society classification: an analysis based on prospectively collected parameters. Endocrine-related cancer, 2010. 17(4): p. 909-18.

6. Rault-Petit, B., et al., Current Management and Predictive Factors of Lymph Node Metastasis of Appendix Neuroendocrine Tumors: A National Study from the French Group of Endocrine Tumors (GTE). Ann Surg, 2019. 270(1): p. 165-171.

7. G, d.L., et al., Surgical Management of Neuroendocrine Tumors of the Appendix in Children and Adolescents: A Retrospective French Multicenter Study of 114 Cases. Pediatric blood \& cancer, 2016. 63(4): p. 598-603.

8. $\mathrm{O}, \mathrm{H}$., et al., Neuroendocrine tumor epidemiology: contrasting Norway and North America. Cancer, 2008. 113(10): p. 2655-64.

9. U, P., et al., Consensus guidelines for the management of patients with digestive neuroendocrine tumours: well-differentiated tumour/carcinoma of the appendix and goblet cell carcinoma. Neuroendocrinology, 2008. 87(1): p. 20-30.

10. L, L., C. J, and D. O, Appendiceal neuroendrocrine neoplasms: incidentaloma or something we should worry about? Current opinion in gastroenterology, 2020. 36(1): p. 48-54.

11. NCCN Clinical Practice Guidelines in Oncology: Neuroendocrine and Adrenal Tumors. Version 2.2020 - July 24, 2020. 
12. A, C., et al., Appendiceal Neuroendocrine Tumors: Does Colon Resection Improve Outcomes? Journal of gastrointestinal surgery : official journal of the Society for Surgery of the Alimentary Tract, 2019.

13. H, E., et al., Survival after resection of appendiceal carcinoma by hemicolectomy and less radical than hemicolectomy: a population-based propensity score matched analysis. Colorectal disease : the official journal of the Association of Coloproctology of Great Britain and Ireland, 2017. 19(10): p. 895-906.

14. Guzman, C., et al., Appendiceal Carcinoid Tumors: Is There a Survival Advantage to Colectomy over Appendectomy? J Gastrointest Surg, 2019.

15. A, C., et al., Propensity adjusted appraisal of the surgical strategy for appendiceal carcinoids. Techniques in coloproctology, 2015. 19(1): p. 35-41.

16. A, M.S., et al., Regional lymph node involvement and outcomes in appendiceal neuroendocrine tumors: a SEER database analysis. Oncotarget, 2017. 8(59): p. 99541-99551.

17. ID, N., et al., The 2019 WHO classification of tumours of the digestive system. Histopathology, 2020. 76(2): p. 182-188.

18. C, M., et al., Novel nomogram combining depth of invasion and size can accurately predict the risk for regional nodal metastases for appendiceal neuroendocrine tumors (A-NET). Journal of surgical oncology, 2017. 116(6): p. 651-657.

19. J, W., et al., Impact of nonappendiceal cancer-specific death on overall survival: a competing risk analysis. Future oncology (London, England), 2019. 15(35): p. 4083-4093.

20. Ho, D.E., et al., Matching as Nonparametric Preprocessing for Reducing Model Dependence in Parametric Causal Inference. Political Analysis, 2007. 15(3): p. 199-236.

21. Austin, P.C., Balance diagnostics for comparing the distribution of baseline covariates between treatment groups in propensity-score matched samples. Statistics in Medicine, 2009. 28(25): p. 3083-3107.

22. C, H., et al., Varying malignant potential of appendiceal neuroendocrine tumors: importance of histologic subtype. Journal of surgical oncology, 2013. 107(2): p. 136-43.

23. Wu, J.J., et al., Impact of nonappendiceal cancer-specific death on overall survival: a competing risk analysis. FUTURE ONCOLOGY, 2019. 15(35): p. 4083-4093.

24. Putter, H., M. Fiocco, and R.B. Geskus, Tutorial in biostatistics: competing risks and multi-state models. Statistics in medicine, 2007. 26(11): p. 2389-2430.

25. Berry, S.D., et al., Competing Risk of Death: An Important Consideration in Studies of Older Adults: COMPETING RISK OF DEATH IN STUDIES OF OLDER ADULTS. Journal of the American Geriatrics Society, 2010. 58(4): p. 783-787.

26. K, D., et al., The risk of lymph node metastases and their impact on survival in patients with appendiceal neuroendocrine neoplasms: a systematic review and meta-analysis of adult and paediatric patients. Endocrine, 2020. 67(1): p. 20-34. 
27. A, C., et al., Clinical Significance of Metastatic Lymph Nodes in the Gut of Patients with Pure and Mixed Primary Appendiceal Carcinoids. Diseases of the colon and rectum, 2016. 59(6): p. 508-12.

28. CG, M., et al., Carcinoid tumor of the appendix: treatment and prognosis. The New England journal of medicine, 1987. 317(27): p. 1699-701.

29. ZM, B. and B. DL, Is right hemicolectomy for 2.0-cm appendiceal carcinoids justified? Archives of surgery (Chicago, III. : 1960), 2006. 141(4): p. 349-52; discussion 352.

30. Breugom, A., et al., Association Between the Most Frequent Complications After Surgery for Stage I-III Colon Cancer and Short-Term Survival, Long-Term Survival, and Recurrences. Annals of surgical oncology, 2016. 23(9): p. 2858-65.

\section{Tables}

Table 1. Characteristics of patients in the LRHC group and RHCM group. 


\begin{tabular}{|c|c|c|c|}
\hline & LRHC & RHCM & P-value \\
\hline $\mathrm{N}$ & 643 & 245 & \\
\hline Age (year, IQR) & $40(27-53)$ & $48(33-58)$ & $<0.01$ \\
\hline \multicolumn{4}{|l|}{ Gender } \\
\hline Female (\%) & $381(59.25)$ & $156(63.67)$ & 0.26 \\
\hline Male (\%) & $262(40.75)$ & $89(36.33)$ & \\
\hline \multicolumn{4}{|l|}{ Race } \\
\hline White (\%) & $562(87.40)$ & $203(82.86)$ & $<0.01$ \\
\hline Black (\%) & $48(7.47)$ & $35(14.29)$ & \\
\hline Other (\%) & $33(5.13)$ & $7(2.86)$ & \\
\hline \multicolumn{4}{|l|}{ Histological type } \\
\hline Pure (\%) & $594(92.38)$ & $208(84.90)$ & $<0.01$ \\
\hline Mixed (\%) & $49(7.62)$ & $37(15.10)$ & \\
\hline \multicolumn{4}{|l|}{ Differentiation } \\
\hline Well differentiated (\%) & $534(83.05)$ & $191(77.96)$ & 0.10 \\
\hline Moderately differentiated (\%) & 109 (16.95) & $54(22.04)$ & \\
\hline \multicolumn{4}{|l|}{ Size $(\mathrm{mm})$} \\
\hline$\leq 10(\%)$ & $439(68.27)$ & $54(22.04)$ & $<0.01$ \\
\hline $11-20(\%)$ & $141(21.93)$ & $77(31.43)$ & \\
\hline$>21(\%)$ & $63(9.80)$ & $114(46.53)$ & \\
\hline \multicolumn{4}{|l|}{ Depth of invasion } \\
\hline LP (\%) & $107(16.64)$ & $14(5.71)$ & $<0.01$ \\
\hline MP/TMP (\%) & $446(69.36)$ & $144(58.78)$ & \\
\hline TS (\%) & $90(14.00)$ & $87(35.51)$ & \\
\hline \multicolumn{4}{|l|}{ Nodal status } \\
\hline NLNE (\%) & $508(79.00)$ & $20(8.16)$ & $<0.01$ \\
\hline LNN (\%) & 105 (16.33) & $143(58.37)$ & \\
\hline LNP (\%) & $30(4.67)$ & $82(33.47)$ & \\
\hline \multicolumn{4}{|l|}{ Metastatic status } \\
\hline No $(\%)$ & 637 (99.07) & $234(95.51)$ & $<0.01$ \\
\hline Yes (\%) & $6(0.93)$ & $11(4.49)$ & \\
\hline
\end{tabular}

Abbreviations: LRHC: less extended than right hemicolectomy; RHCM: right hemicolectomy/ more extended procedure; LP: invasion of the lamina propria; MP/TMP: invasion or through the muscularis propria; TS: invasion through the serosa and adjacent structures; NLNE: no lymph nodes examined; LNN: lymph nodes examined with negative lymph nodes; LNP: lymph nodes examined with positive lymph nodes.

Table 2. Associations of demographic and clinical characteristics with cancer-specific death. 


\begin{tabular}{|c|c|c|c|c|c|c|}
\hline \multirow[b]{2}{*}{ Factor } & \multicolumn{3}{|c|}{ Univariate Analyses } & \multicolumn{3}{|c|}{ Multivariate Analysis } \\
\hline & SHR & 95\% CI & $P$ value & SHR & $95 \%$ CI & $P$ value \\
\hline Age (year) & 1.07 & $1.03-1.10$ & $<0.01$ & 1.05 & $0.99-1.10$ & 0.10 \\
\hline \multicolumn{7}{|l|}{ Gender } \\
\hline Female & Reference & & & & & \\
\hline Male & 1.23 & $0.44-3.44$ & 0.69 & & & \\
\hline \multicolumn{7}{|l|}{ Race } \\
\hline White & Reference & & & & & \\
\hline Black & 1.62 & $0.37-7.14$ & 0.52 & & & \\
\hline Other & 4.34 & 0.98-19.19 & 0.05 & & & \\
\hline \multicolumn{7}{|l|}{ Histological type } \\
\hline Pure & Reference & & & Reference & & \\
\hline Mixed & 17.10 & $5.37-54.30$ & $<0.01$ & 16.95 & $2.72-105.71$ & $<0.01$ \\
\hline \multicolumn{7}{|l|}{ Differentiated grade } \\
\hline Well differentiated & Reference & & & Reference & & \\
\hline Moderately differentiated & 3.85 & $1.33-11.10$ & 0.01 & 0.48 & $0.07-3.18$ & 0.44 \\
\hline \multicolumn{7}{|l|}{ Size $(\mathrm{mm})$} \\
\hline$\leq 10$ & Reference & & & Reference & & \\
\hline $11-20$ & 3.51 & $0.32-38.70$ & 0.31 & 1.02 & $0.09-11.26$ & 0.99 \\
\hline$>21$ & 20.13 & $2.56-158.40$ & $<0.01$ & 2.28 & 0.13-39.94 & 0.57 \\
\hline \multicolumn{7}{|l|}{ Depth of invasion ${ }^{a}$} \\
\hline MP/TMP & Reference & & & Reference & & \\
\hline TS & 3.73 & $1.24-11.20$ & 0.02 & 0.55 & $0.14-2.14$ & 0.39 \\
\hline \multicolumn{7}{|l|}{ Nodal status } \\
\hline LNN & Reference & & & Reference & & \\
\hline LNP & 2.92 & $0.84-10.11$ & 0.09 & 3.67 & $0.84-16.12$ & 0.09 \\
\hline NLNE & 0.42 & 0.09-1.93 & 0.27 & 1.33 & $0.12-14.45$ & 0.82 \\
\hline \multicolumn{7}{|l|}{ Metastatic status } \\
\hline No & Reference & & & Reference & & \\
\hline Yes & 27.20 & $9.48-77.80$ & $<0.01$ & 9.18 & $2.07-40.79$ & $<0.01$ \\
\hline \multicolumn{7}{|l|}{ Surgical procedure } \\
\hline LRHC & Reference & & & Reference & & \\
\hline RHCM & 2.65 & $0.92-7.64$ & 0.07 & 1.77 & $0.40-7.85$ & 0.45 \\
\hline
\end{tabular}

Abbreviations: SHR: Subdistribution hazard ratio; CI: Confidential interval; LRHC: less extended than right hemicolectomy; RHCM: right hemicolectomy/ more extended procedure; LP: invasion of the lamina propria; MP/TMP: invasion or through the muscularis propria; TS: invasion through the serosa and adjacent structures; NLNE: no lymph nodes examined; LNN: lymph nodes examined with negative lymph nodes; LNP: lymph nodes examined with positive lymph nodes.

a. Patients with invasion of LP were excluded from univariate and multivariate analyses because none of them dead of cancer-specific death, and analyses could not be performed in such a situation.

Table 3. Characteristics of patients in the LRHC group and RHCM group after propensity score matching. 


\begin{tabular}{|c|c|c|c|}
\hline & LRHC (\%) & RHCM (\%) & P-value \\
\hline Number & 136 & 136 & \\
\hline Age (year, IQR) & $46.18(16.09)$ & $45.18(15.50)$ & 0.60 \\
\hline \multicolumn{4}{|l|}{ Gender } \\
\hline Female & $81(59.56)$ & $84(61.76)$ & 0.80 \\
\hline Male & $55(40.44)$ & $52(38.24)$ & \\
\hline \multicolumn{4}{|l|}{ Race } \\
\hline White & $115(84.56)$ & $110(80.88)$ & 0.72 \\
\hline Black & $18(13.24)$ & $22(16.18)$ & \\
\hline Other & $3(2.21)$ & $4(2.94)$ & \\
\hline \multicolumn{4}{|l|}{ Histological type } \\
\hline Pure & $115(84.56)$ & $117(86.03)$ & 0.86 \\
\hline Mixed & $21(15.44)$ & 19 (13.97) & \\
\hline \multicolumn{4}{|l|}{ Differentiation } \\
\hline Well differentiated & $103(75.74)$ & $100(73.53)$ & 0.78 \\
\hline Moderately differentiated & $33(24.26)$ & $36(26.47)$ & \\
\hline \multicolumn{4}{|l|}{ Size $(\mathrm{mm})$} \\
\hline$\leq 10$ & $51(37.50)$ & $51(37.50)$ & 0.75 \\
\hline $11-20$ & $45(33.09)$ & $40(29.41)$ & \\
\hline$>21$ & $40(29.41)$ & $45(33.09)$ & \\
\hline \multicolumn{4}{|l|}{ Depth of invasion } \\
\hline LP & $15(11.03)$ & $12(8.82)$ & 0.77 \\
\hline MP/TMP & $81(59.56)$ & $80(58.82)$ & \\
\hline TS & $40(29.41)$ & $44(32.35)$ & \\
\hline \multicolumn{4}{|l|}{ Nodal status } \\
\hline NLNE & $88(64.71)$ & 79 (58.09) & 0.42 \\
\hline LNN & 28 (20.59) & $37(27.21)$ & \\
\hline LNP & $20(14.71)$ & $20(14.71)$ & \\
\hline \multicolumn{4}{|l|}{ Metastatic status } \\
\hline No & 133 (97.79) & $131(96.32)$ & 0.72 \\
\hline Yes & $3(2.21)$ & $5(3.68)$ & \\
\hline
\end{tabular}

Abbreviations: LRHC: less extended than right hemicolectomy; RHCM: right hemicolectomy/ more extended procedure; LP: invasion of the lamina propria; MP/TMP: invasion or through the muscularis propria; TS: invasion through the serosa and adjacent structures; NLNE: no lymph nodes examined; LNN: lymph nodes examined with negative lymph nodes; LNP: lymph nodes examined with positive lymph nodes.

\section{Figures}




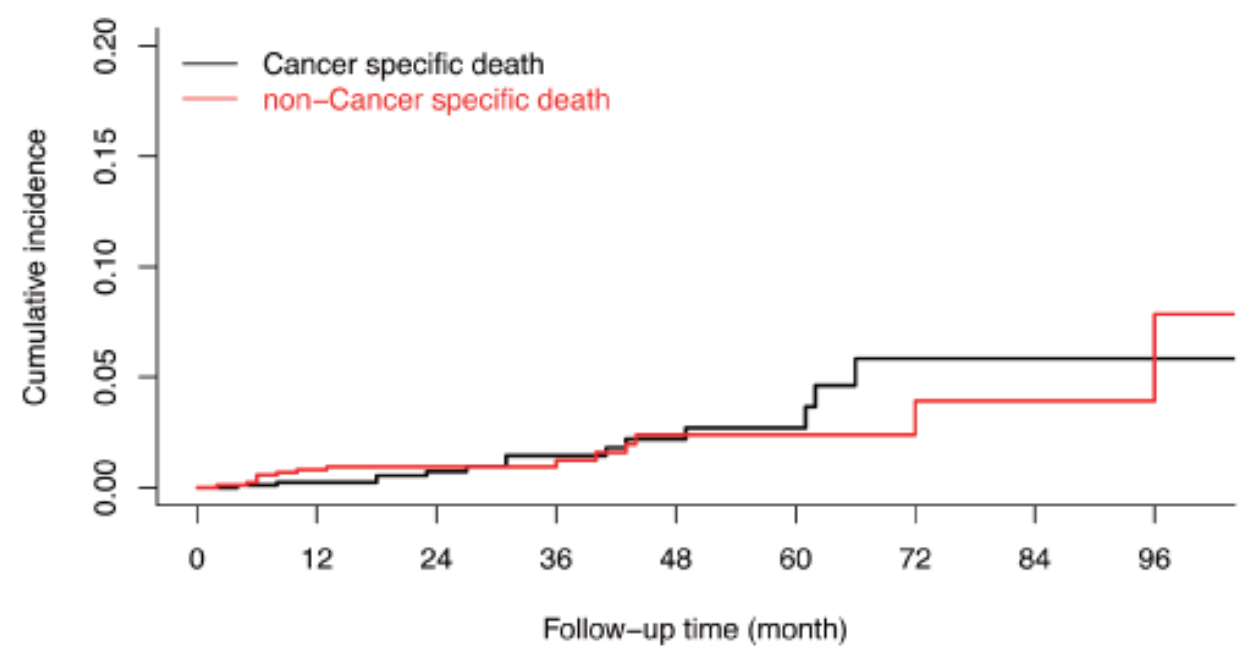

Figure 1

Cumulative incidences of cancer specific death and non-cancer specific death. Patients undergoing RHCM have a higher incidence of CSD than those undergoing LRHC $(P=0.04)$. 


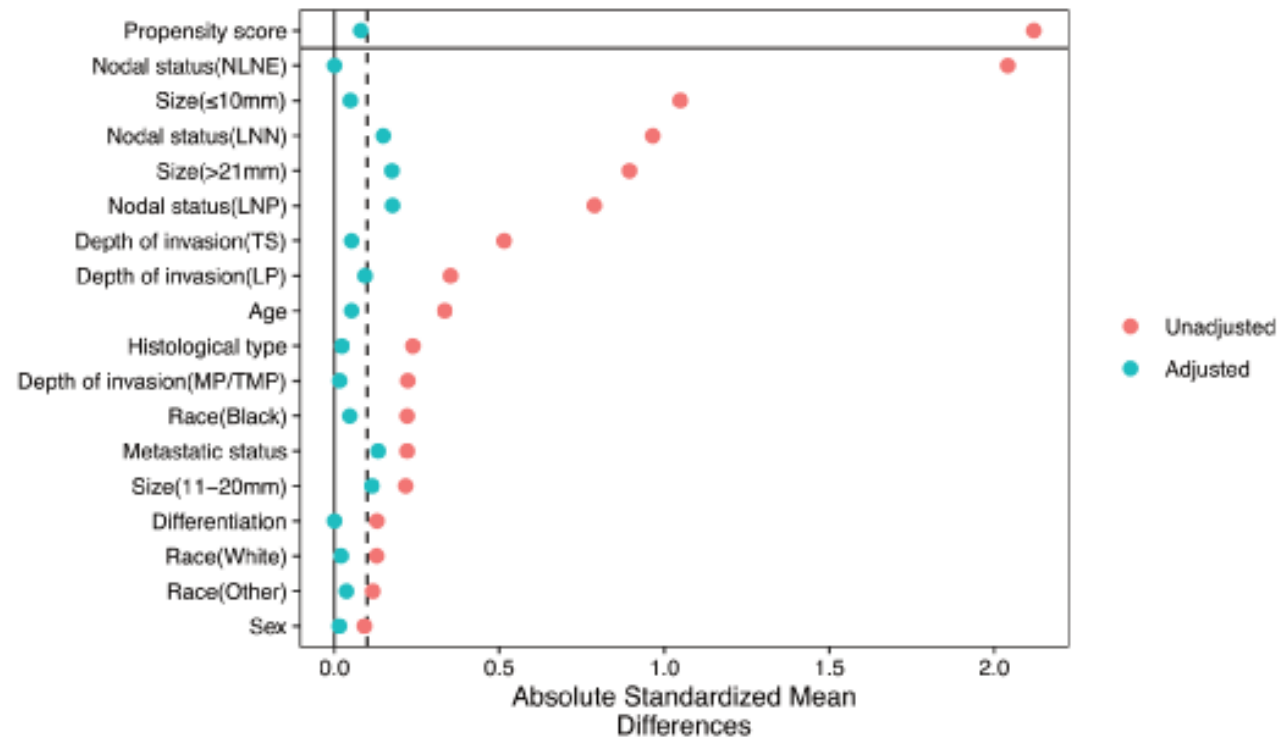

Figure 2

"Forest plot" that presents the comparison of LRHC and RHCM in the entire cohort and subgroups. a. Patients in some subgroups cannot be compared because no patient dead from cancer specific death in the LRHC or RHCM group, and the Gray test cannot performed in such situations. Abbreviations: LRHC: less extended than right hemicolectomy; RHCM: right hemicolectomy/ more extended procedure; LP: invasion of the lamina propria; MP/TMP: invasion or through the muscularis propria; TS: invasion through the serosa and adjacent structures; NLNE: no lymph nodes examined; LNN: lymph nodes examined with negative lymph nodes; LNP: lymph nodes examined with positive lymph nodes.

\section{Supplementary Files}

This is a list of supplementary files associated with this preprint. Click to download.

- AppendixFigure1.jpg 
- AppendixFigure2.jpg

- AppendixTable1.docx 